\title{
Efecto en la respuesta temporal del parámetro ajustable del control adaptativo con modelo de referencia de primer orden
}

\section{RESUMEN}

Se presentan los resultados experimentales del control adaptativo con modelo de referencia, de una planta que de acuerdo a su identificación previamente realizada se puede representar como un sistema de primer orden. Se elige como modelo de referencia también uno de primer orden con ganancia unitaria. Se realiza el análisis del controlador adaptativo, justificándose que su desempeño en lazo cerrado dependerá de la elección adecuada de un parámetro escalar ajustable. Se implementa el controlador y se registran las respuestas temporales de la planta para entradas tipo escalón para diferentes valores del parámetro ajustable.

Palabras clave: control adaptativo, gradiente del error, modelo de referencia, sobreimpulso, tiempo de establecimiento

Effect on Temporal Response Adjustable Parameter Adaptive Control with Reference Model First Order

\section{ABSTRACT}

Experimental results are presented adaptive control with reference model of a plant according to your previously made identification can be represented as a first order system. Is chosen as the reference model also one of the first order with unity gain. Adaptive controller analysis is performed, justifying its performance in closed loop depends on the proper choice of a scale parameter adjustable. The controller is implemented and temporary plant responses to step inputs for different kinds of adjustable parameter values are recorded.

Keywords: adaptive control, model reference gradient error, overshoot, settling time

\section{INTRODUCCIÓN}

El control adaptativo es un controlador no lineal en el que el estado del proceso puede ser separado en dos escalas de tiempo que evolucionan a diferente velocidad. La escala lenta corresponde a los cambios de los parámetros y la rápida corresponde a la dinámica del bucle ordinario de realimentación [1]. Los sistemas adaptativos con modelo de referencia fueron diseñados primeramente para sistemas continuos por minimización de un índice de actuación, siendo ese índice la integral del error al cuadrado [2]. Esta regla de diseño fue propuesta por Whitaker del MIT en 1958, por eso se denomina regla del MIT.

Se presenta la deducción de esta regla para el caso del control de una planta de primer orden que se desea siga la respuesta temporal de un sistema de referencia de primer orden también.

Aquí se demuestra, que finalmente se tiene un parámetro ajustable que es la ganancia de adaptación, la cual es la gobierna la respuesta temporal del sistema de lazo cerrado. Este parámetro de adaptación debe ser cuidadosamente elegido.

Es nuestro interés, comprobar el desempeño en lazo cerrado de los controladores adaptativos con modelo de referencia de primer orden mediante la variación del parámetro ajustable.

\section{DISEÑO DEL CONTROLADOR ADAPTATIVO CON MODE- LO DE REFERENCIA POR EL MÉTODO DEL GRADIENTE}

Sea una planta a ser controlada, para la cual la señal de entrada es $u(t)$ y la señal de salida $y(t)$. Considere que se trata de un sistema de primer orden con función de transferencia $\mathrm{G}(\mathrm{s})$ dada por :

$G(s)=\frac{Y(s)}{U(s)}=\frac{b}{s+a}$

De (1) obtenemos :

$s Y(s)=-a Y(s)+b U(s)$

Doctor en Ingeniería Industrial. Docente Facultad de Ingeniería Electrónica y Eléctrica, Universidad Nacional Mayor de San Marcos, Lima. E-mail: bvargast@unmsm.edu.pe 
Si aplicamos la inversa de Laplace a la relación anterior :

$\dot{y}=-a y+b u$

Sea el modelo de referencia de primer orden, con entrada $r(t)$ y salida $y_{m}(t)$. Ese sistema está representada por la función de transferencia $G_{m}(s)$ :

$$
G_{m}(s)=\frac{Y_{m}(s)}{R(s)}=\frac{b_{m}}{s+a_{m}}
$$

Análogamente a lo que se hizo a partir de (1), la ecuación diferencial que se obtiene a partir de (3) es:

$$
\dot{y}_{m}=-a_{m} y_{m}+b_{m} r
$$

Planteamos el objetivo de obtener mediante una estrategia de control que la salida de la planta $y(t)$ se iguale a la salida del modelo de referencia $y_{m}(t)$, para una entrada $r(t)$ del tipo escalón, es decir :

$y=y_{m}$

si se llega al objetivo, se cumplirá también que :

$\dot{y}=\dot{y}_{m}$

por tanto, igualando (2) y (4), se cumplirá :

$$
u=\frac{a}{b} y-\frac{a_{m}}{b} y_{m}+\frac{b_{m}}{b} r
$$

Considerando el objetivo expresado en (5), de la ecuación anterior se deduce la ley de control para $\mathrm{u}(\mathrm{t})$ que nos llevará al objetivo deseado : $u=\frac{\left(a-a_{m}\right)}{b} y+\frac{b_{m}}{b} r$

Definimos los parámetros de la ley de control (6) como :

$\theta_{1}=\frac{\left(\boldsymbol{a}-\boldsymbol{a}_{\boldsymbol{m}}\right)}{\boldsymbol{b}}$

$\theta_{2}=\frac{\boldsymbol{b}_{\boldsymbol{m}}}{\boldsymbol{b}}$

por tanto, la ecuación (6) para la ley de control, al utilizar (7) y (8) se convierte en :

$\boldsymbol{u}=\theta_{1} \boldsymbol{y}+\theta_{2} \boldsymbol{r}$

Sea el índice de desempeño definido como :

$\boldsymbol{J}=\frac{1}{2} \boldsymbol{e}^{2}$

donde la señal de error e(t) se define como :

$e=y_{m}-y$

Agregamos al objetivo de encontrar la señal de control $u(t)$ que permita que la salida de la planta y $(t)$ siga a la salida de referencia $y_{m}(t)$, la condición que sea así minimizando la función de costo expresada por (10); entonces se requiere que los parámetros $\theta_{1}$ y $\theta_{2}$ desconocidos de la ley de control sean ajustados de una manera que minimice J. Para ello se requiere que esos parámetros se cambien en la dirección negativa del gradiente de la función de costo, esta es conocida como la regla del MIT [3]. Esta regla se representa como :

$\frac{d \theta_{i}}{d t}=-\gamma \frac{d J}{d \theta_{i}}$ 
para $i=1,2$.

De (10) se obtiene :

$\frac{d J}{d \theta_{i}}=e \frac{d e}{d \theta_{i}}$

por tanto, obtenemos de (13) en (12):

$\frac{d \theta_{i}}{d t}=-\gamma e \frac{d e}{d \theta_{i}}$

Considerando que la entrada de referencia $r(t)$ será del tipo escalón, entonces $\mathrm{y}_{\mathrm{m}}(\mathrm{t})$ en estado estacionario será constante, entonces de (11) se deduce :

$d e=-d y$

Utilizando el operador $p=d / d t$ y reemplazando (9) en (2), obtenemos :

$\boldsymbol{y}=\frac{\boldsymbol{b} \theta_{2}}{\boldsymbol{p}+\boldsymbol{a}-\boldsymbol{b} \theta_{1}} \boldsymbol{r}$

a partir de (15) en (14) obtenemos :

$\frac{d \theta_{i}}{d t}=\gamma e \frac{d y}{d \theta_{i}}$

Utilizando (17) con i=1 y utilizando (16) se obtiene:

$\frac{d \theta_{1}}{d \boldsymbol{t}}=\gamma \boldsymbol{e} \frac{\boldsymbol{b} \theta_{2}}{\left(\boldsymbol{p}+\boldsymbol{a}-\boldsymbol{b} \theta_{1}\right)^{2}} \boldsymbol{b r}$

$\frac{d \theta_{1}}{d t}=\gamma \frac{b}{p+a-b \theta_{1}} e y$

Con $\mathrm{i}=2$ en (17) y considerando (16) se obtiene:

$\frac{d \theta_{2}}{d t}=\gamma \frac{b}{p+a-b \theta_{1}} e r$
Con (7) en (18) y (19), se obtiene :

$\frac{d \theta_{1}}{d t}=\gamma \frac{b}{p+a-b \theta_{1}} e y$

$\frac{d \theta_{2}}{d t}=\gamma \frac{b}{p+a_{m}} e r$

El parámetro y se ajustará según convenga; se puede considerar que :

$\alpha \boldsymbol{b}_{m}=\gamma \boldsymbol{b}$

donde ahora el parámetro variable sería $\alpha$. Este parámetro corresponde a una ganancia ajustable que determinará la respuesta temporal del sistema de lazo cerrado.

Por tanto (20) y (21) se convierten en :

$\frac{d \theta_{1}}{d t}=\gamma \frac{b}{p+a_{m}} e y$

$\frac{d \theta_{2}}{d t}=\alpha \frac{b_{m}}{p+a_{m}} e r$

La figura 1, representa la implementación de la ley de control (9), mediante la integración de las ecuaciones (22) y (23).

Figura 1. Diagrama de bloques de obtención de la ley de control.

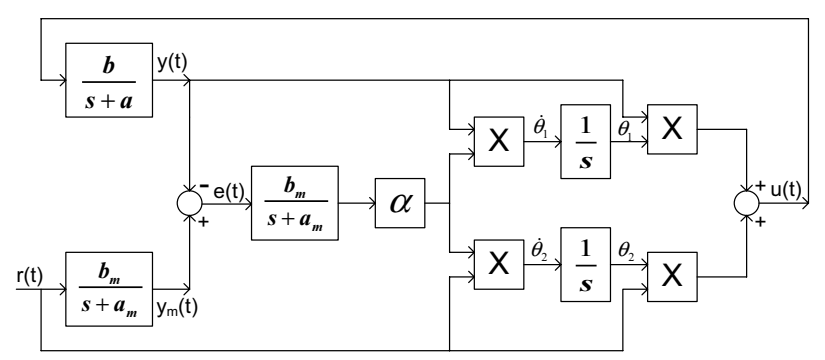

Fuente: Elaboración propia. 


\section{METODOLOGÍA EMPLEADA}

\section{A. Identificación analógica de la planta}

La planta a ser controlada fue identificada previamente. Para obtener el modelo de función de transferencia $\mathrm{G}(\mathrm{s})$ que represente las variaciones de salida por efecto de las variaciones de entrada de la planta; se aplica en lazo abierto una señal de entrada, la cual produce los cambios en la salida. Se han registrado mediante un sistema de adquisición de datos, las señales de entrada y salida de la planta que se muestran en las figuras $2 \mathrm{a}$ y $2 \mathrm{~b}$ respectivamente.

Figura 2a. Señal del tipo cambio escalón aplicada en la entrada de la planta.

SEÑAL DE ENTRADA APLICADA A LA PLANTA

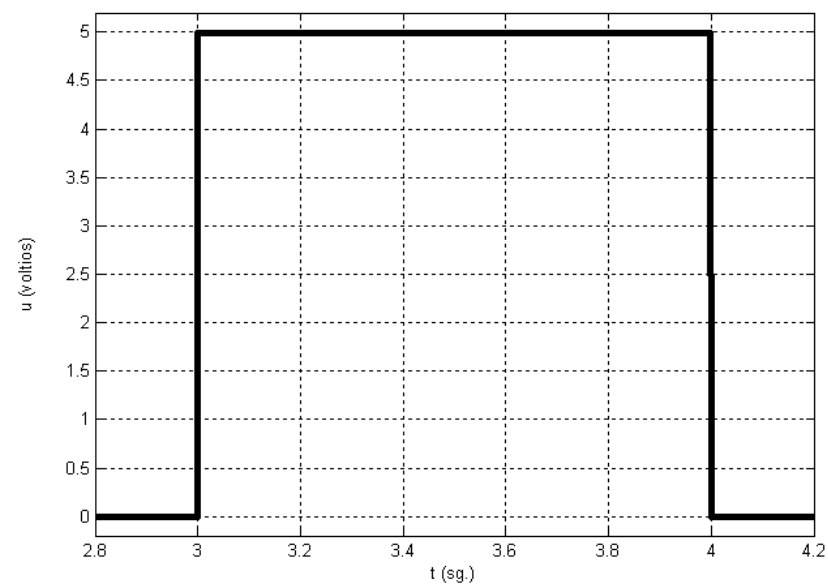

Figura 2b. Respuesta de la planta para un cambio escalón en la entrada de la planta de prueba.



Fuente: Elaboración propia.
La figura 2b. es la llamada curva de reacción del proceso a controlar. En base a los resultados de esa prueba, desarrollamos la identificación analógica de la planta utilizando el método de los dos puntos de Alfaro [4] y obtenemos un modelo representativo de primer orden. El modelo de la planta obtenido es el siguiente :

$$
G(s)=\frac{47.04}{\mathrm{~s}+31.851}
$$

Para validar el modelo se aplican señales de entrada diferentes a la planta, se registran sus respuesta. La respuesta del modelo para esas mismas entradas se comparan con las medidas, obteniéndose una gran aproximación en ambas respuestas. La figura 3 , muestra para un caso la respuesta medida y la respuesta del modelo, se observa que ambas están prácticamente superpuestas.

Figura 3. Respuesta del modelo y del proceso para la misma entrada de prueba (validación del modelo identificado).

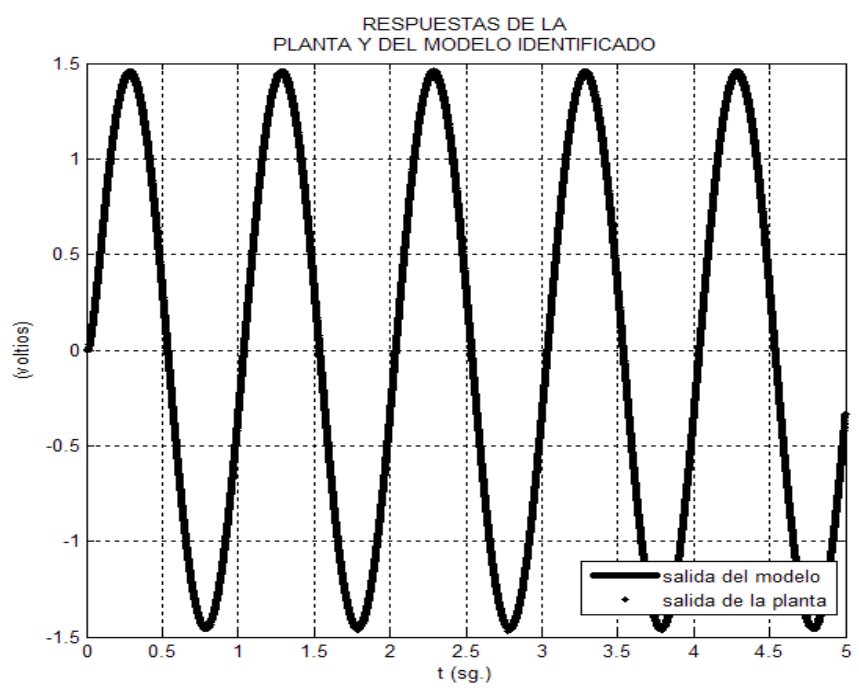

Fuente: Elaboración propia.

\section{B. Implementación del controlador adaptativo y del sistema de lazo cerrado}

El controlador adaptativo cuyo diagrama de bloques se muestra en la figura 1 , se implementó utilizando como base el sistema de adquisición de datos basado en la tarjeta DAQ USB 6215 de National Instruments. En todos los casos el modelo 
de referencia utilizado fue configurado para una ganancia unitaria. Se hicieron pruebas con varias constantes de tiempo del modelo de referencia de primer orden, eligiéndose en función de la constante de tiempo de la planta identificada. Se eligieron factores $\beta$ de $1,0.5,0.25,2$ y 4 veces, donde denominamos $\beta$ a la relación entre el polo del modelo de referencia de primer orden y el polo del modelo de la planta identificada.

\section{RESULTADOS}

En las tablas I, II, II, IV y V de los Anexos, se muestran los resultados de los parámetros de la respuesta temporal de la planta, cuando es controlada por el controlador adaptativo con modelo de referencia de primer orden implementado de acuerdo al diagrama de bloques de la figura 1. Corresponden a pruebas del sistema de lazo cerrado, para entradas del tipo escalón. Cada tabla corresponde a un valor de $\beta$ diferente. De cada respuesta, se han medido los valores de tiempo de establecimiento, porcentaje de sobreimpulso y error de estado estacionario.

También en los Anexos, se muestran en las figuras de $\mathrm{A} 1$ a $\mathrm{A} 5$ las respuestas temporales para las cuales se han obtenido los mejores parámetros de respuesta temporal de acuerdo al valor de $\beta$ elegido.
La variación de la señal de control, se muestran en las figuras de A6 a A10 de los Anexos, para cada una de las respuestas temporales con los mejores parámetros de respuesta temporal de acuerdo al valor de $\beta$ elegido.

\subsection{REFERENCIAS}

[1] Francisco Rodríguez, Manuel López M. Control Adaptativo y Robusto. Universidad de Sevilla, 1996.

[2] C.C. Hang and P.C Parks. Comparatve Studies of Model Reference Adaptative Control Systems. IEEE T.A.C. Vol AC 18-5 pp 419-428.

[3] Javier Perez, Roger Pelayo R, Francisco Manzanarez. Implementación de un controlador adaptativo para un sistema de primer orden con ganancia y polo variantes, basado en DSP. RIEE\&C, Revista de Ingeniería Eléctrica, Electrónica y Computación, Vol. 2 No. 1, Diciembre 2006, Instituto Tecnológico de Sonora.

[4] Víctor Alfaro. Identificación de Procesos Sobreamortiguados Utilizando Técnicas de Lazo Abierto. Ingeniería, 1, 11-25, 2001. 


\section{ANEXOS}

\section{TABLA I}

Resultados Experimentales de Respuesta Temporal del Control Adaptativo con Modelo de Referencia para $\beta=1$

\begin{tabular}{|c|c|c|c|}
\hline \multicolumn{4}{|c|}{$\boldsymbol{\beta = 1}$} \\
\hline $\begin{array}{c}\text { Ganancia } \\
\text { ajustable } \alpha\end{array}$ & $\begin{array}{c}\text { Tiempo de } \\
\text { establecimiento } \\
\text { ts (sg.) }\end{array}$ & $\begin{array}{c}\text { Sobreimpulso MP } \\
(\%)\end{array}$ & $\begin{array}{c}\text { Error de estado } \\
\text { estacionario ess }\end{array}$ \\
\hline 0.5 & 0.70 & 0.00 & 0 \\
\hline 1 & 0.55 & 4.00 & 0 \\
\hline 5 & 0.75 & 9.00 & 0 \\
\hline 10 & 0.70 & 2.00 & 0 \\
\hline 100 & 0.50 & 16.70 & 0 \\
\hline
\end{tabular}

Fuente: Elaboración propia.

TABLA II

Resultados Experimentales de Respuesta Temporal del Control Adaptativo con Modelo de Referencia para $\beta=0.25$

\begin{tabular}{|c|c|c|c|}
\hline \multicolumn{5}{|c|}{$\boldsymbol{\beta = 0 . 2 5}$} \\
\hline $\begin{array}{c}\text { Ganancia } \\
\text { ajustable } \\
\boldsymbol{\alpha}\end{array}$ & $\begin{array}{c}\text { Tiempo de } \\
\text { establecimiento } \\
\text { ts (sg.) }\end{array}$ & $\begin{array}{c}\text { Sobreimpulso } \\
\text { MP (\%) }\end{array}$ & $\begin{array}{c}\text { Error de estado } \\
\text { estacionario } \\
\text { ess }\end{array}$ \\
\hline 0.25 & 2.70 & 0.00 & 0 \\
\hline 0.5 & 2.00 & 0.00 & 0 \\
\hline 1 & 1.70 & 2.00 & 0 \\
\hline 5 & 3.00 & 22.56 & 0 \\
\hline 10 & 2.33 & 27.93 & 0 \\
\hline 100 & 1.85 & 41.60 & 0 \\
\hline
\end{tabular}

Fuente: Elaboración propia.

\section{TABLA III}

Resultados Experimentales de Respuesta Temporal del Control Adaptativo con Modelo de Referencia para $\beta=0.5$

\begin{tabular}{|l|l|l|l|}
\hline \multicolumn{2}{|c|}{$\beta=0.5$} \\
$\begin{array}{c}\text { Ganancia } \\
\text { ajustable } \\
\alpha\end{array}$ & $\begin{array}{c}\text { Tiempo de } \\
\text { establecimiento } \\
\text { ts (sg.) }\end{array}$ & $\begin{array}{c}\text { Sobreimpulso } \\
\text { MP (\%) }\end{array}$ & $\begin{array}{c}\text { Error de estado } \\
\text { estacionario } \\
\text { ess }\end{array}$ \\
\hline 0.25 & 2.00 & 0.00 & 0 \\
\hline 0.5 & 1.00 & 0.00 & 0 \\
\hline 1 & 0.70 & 0.00 & 0 \\
\hline
\end{tabular}




\begin{tabular}{|l|l|l|l|}
\hline 5 & 1.33 & 6.40 & 0 \\
\hline 10 & 1.43 & 14.20 & 0 \\
\hline 100 & 0.80 & 22.56 & 0 \\
\hline
\end{tabular}

Fuente: Elaboración propia.

\section{TABLA IV}

Resultados Experimentales de Respuesta Temporal del Control Adaptativo con Modelo de Referencia para $\beta=2$

\begin{tabular}{|c|c|c|c|}
\hline \multicolumn{4}{|c|}{$\boldsymbol{\beta = 2}$} \\
\hline $\begin{array}{c}\text { Ganancia } \\
\text { ajustable } \\
\boldsymbol{\alpha}\end{array}$ & $\begin{array}{c}\text { Tiempo de } \\
\text { establecimiento } \\
\mathbf{t}_{\mathbf{s}} \text { (sg.) }\end{array}$ & $\begin{array}{c}\text { Sobreimpulso } \\
\mathbf{M}_{\mathbf{p}}(\%)\end{array}$ & $\begin{array}{c}\text { Error de estado } \\
\text { estacionario } \\
\mathbf{e}_{\mathbf{s s}}\end{array}$ \\
\hline 0.5 & 0.90 & 4.00 & 0 \\
\hline 1 & 0.57 & 3.50 & 0 \\
\hline 10 & 0.20 & 2.00 & 0 \\
\hline 50 & 0.23 & 4.90 & 0 \\
\hline 100 & 0.21 & 10.35 & 0 \\
\hline 200 & 0.14 & 30.37 & 0 \\
\hline
\end{tabular}

Fuente: Elaboración propia.

\section{TABLA V}

Resultados Experimentales de Respuesta Temporal del Control Adaptativo con Modelo de Referencia para $\beta=4$

\begin{tabular}{|c|c|c|c|}
\hline \multicolumn{4}{|c|}{$\boldsymbol{\beta = 4}$} \\
\hline $\begin{array}{c}\text { Ganancia } \\
\text { ajustable } \\
\boldsymbol{\alpha}\end{array}$ & $\begin{array}{c}\text { Tiempo de } \\
\text { establecimiento } \\
\mathbf{t}_{\mathbf{s}} \text { (sg.) }\end{array}$ & $\begin{array}{c}\text { Sobreimpulso } \\
\mathbf{M}_{\mathbf{p}}(\%)\end{array}$ & $\begin{array}{c}\text { Error de estado } \\
\text { estacionario } \\
\mathbf{e}_{\mathrm{ss}}\end{array}$ \\
\hline 0.5 & 0.90 & 4.00 & 0 \\
\hline 1 & 0.60 & 6.45 & 0 \\
\hline 10 & 0.29 & 17.68 & 0 \\
\hline 50 & 0.16 & 12.79 & 0 \\
\hline 100 & 0.11 & 15.72 & 0 \\
\hline 150 & 0.095 & 22.00 & 0 \\
\hline
\end{tabular}

Fuente: Elaboración propia. 
Figura A1. Respuesta temporal de la planta con las mejores características, obtenido para $\beta=1$ $(\alpha=1)$.

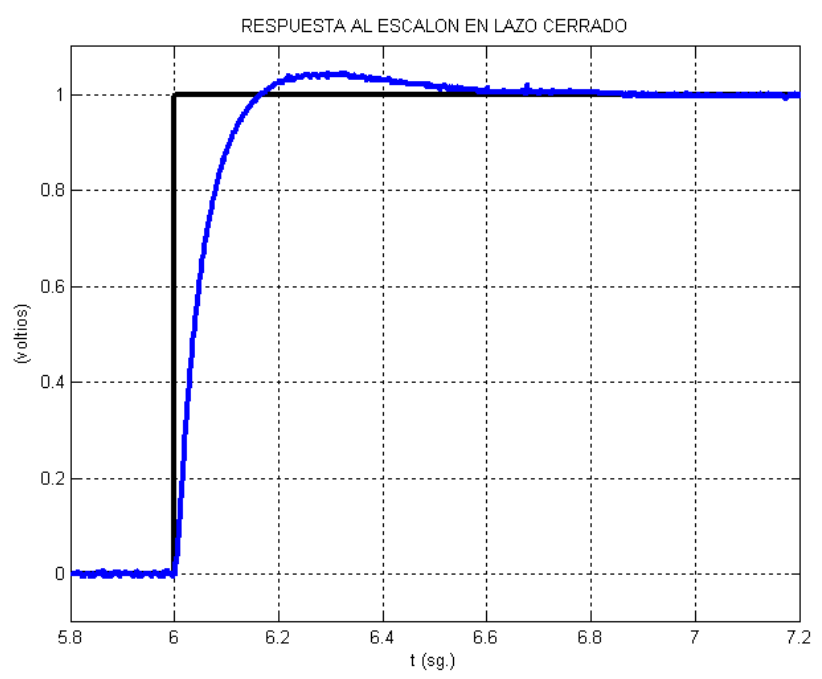

Fuente: Elaboración propia.

Figura A2. Respuesta temporal de la planta con las mejores características, obtenido para $\beta=0.25$ $(\alpha=1)$.

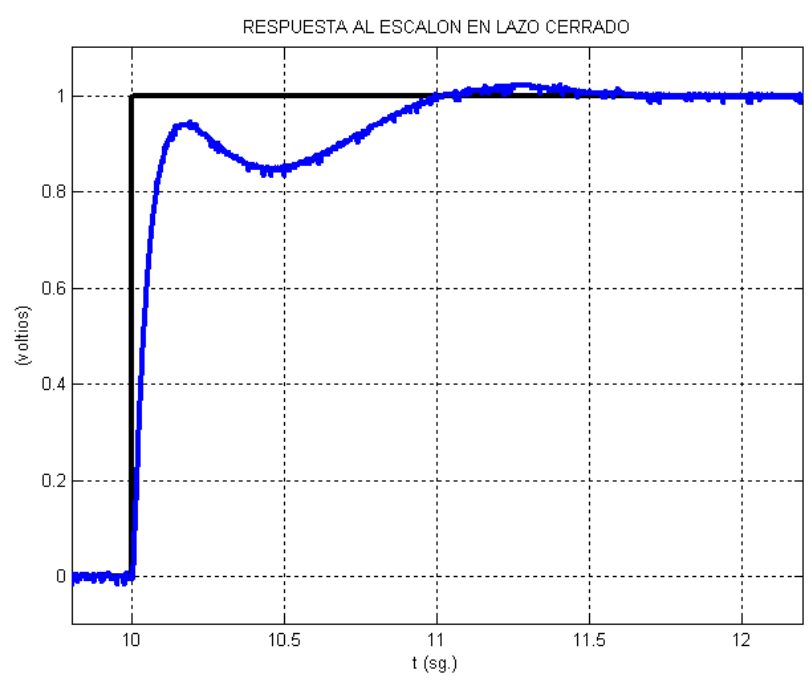

Fuente: Elaboración propia.
Figura A3. Respuesta temporal de la planta con las mejores características, obtenido para $\beta=0.5$ $(\alpha=1)$.

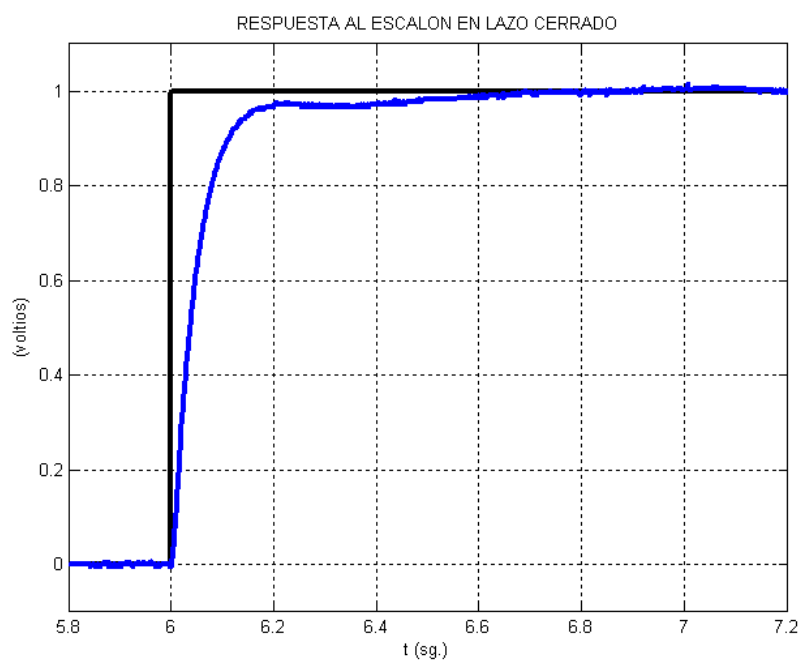

Fuente: Elaboración propia.

Figura A4. Respuesta temporal de la planta con las mejores características, obtenido para $\beta=2$ $(\alpha=10)$.

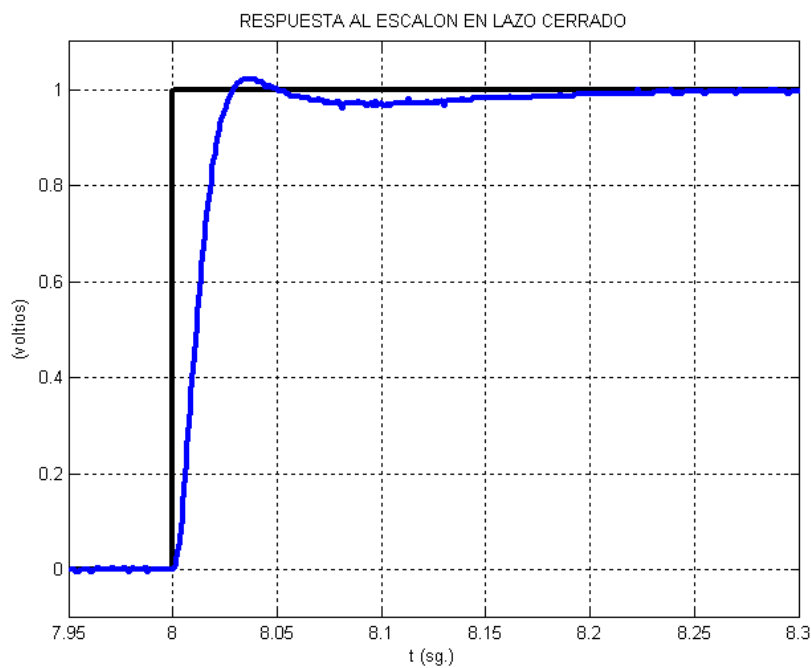

Fuente: Elaboración propia. 
Figura A5. Respuesta temporal de la planta con las mejores características, obtenido para $\beta=4$ $(\alpha=100)$.

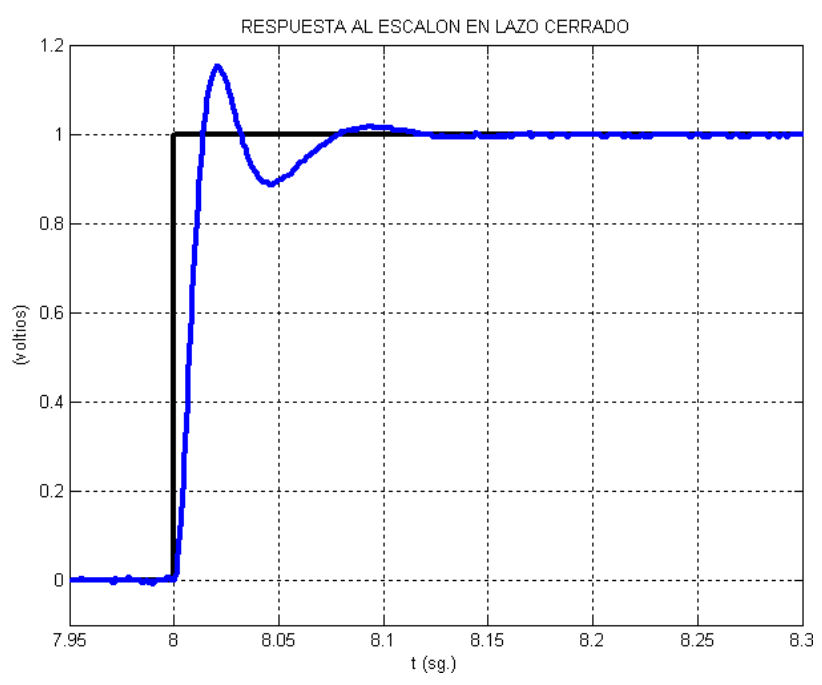

Fuente: Elaboración propia.

Figura A6. Señal de control, obtenida para $\beta=1$

$(\alpha=1)$



Fuente: Elaboración propia.
Figura A7. Señal de control, obtenida para $\beta=0.25(\alpha=1)$.

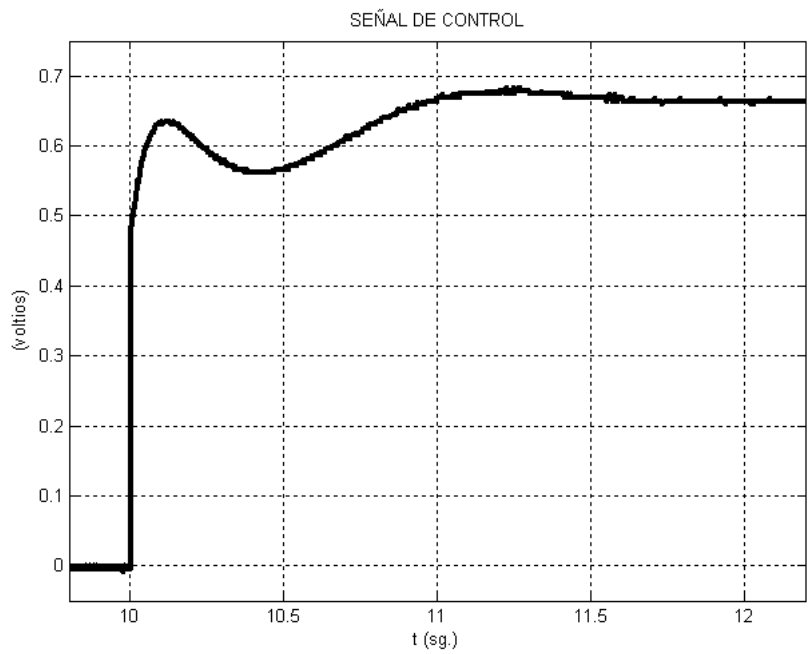

Fuente: Elaboración propia

Figura A8. Señal de control, obtenida para $\beta=0.5$ $(\alpha=1)$.



Fuente: Elaboración propia. 
Figura A9. Señal de control, obtenida para $\beta=2$ $(\alpha=10)$.



Fuente: Elaboración propia.
Figura A10. Señal de control, obtenida para $\beta=4$ $(\alpha=100)$.

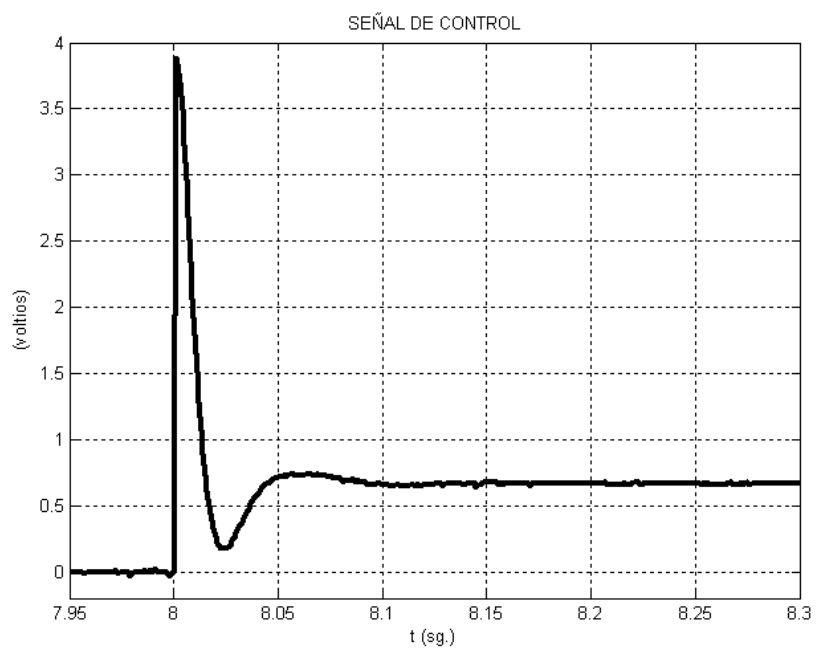

Fuente: Elaboración propia. 